\title{
Effect of different parameters on the tribological performance of polypropylene/thermoplastic polyurethane blends under dry sliding conditions
}

\author{
SONER SAVAŞE \\ Department of Materials Science and Engineering, Engineering Faculty, Erciyes University, 38039 Melikgazi, Kayseri, \\ Turkey \\ ssavas@erciyes.edu.tr
}

MS received 5 February 2018; accepted 2 October 2018; published online 27 March 2019

\begin{abstract}
In this study, polypropylene/thermoplastic polyurethane (PP/TPU) blends (weight ratios of 75/25 and 25/75) were produced by melt blending, and a maleic anhydride-grafted polypropylene (PP-g-MA) copolymer was used as a coupling agent. The effect of the concentration of the coupling agent (5-11 phr), TPU concentration (25-75\%), normal load $(2-4 \mathrm{~N})$ and sliding velocity $(60-72 \mathrm{rpm})$ on the wear properties of the blends were characterized through a pin-on-disc abrasive wear test. A $2^{4}$ full-factorial experimental design was chosen to screen the significant factors and their interactions influencing the tribological performance of the blends. After the tests, the morphology of the worn surface of the samples was observed using scanning electron microscopy. Shore D hardness tests were also conducted and the relationships between these behaviours were analysed on the basis of the findings. According to the wear test results, the factors that have the greatest effect on the wear rates are as follows: normal load, TPU concentration, sliding velocity and PP-g-MA concentration, respectively. The wear rate of the blends increases by increasing the normal load, TPU concentration and sliding speed, and slightly decreases by increasing the PP-g-MA concentration. As expected, the wear and hardness results showed the apparent superiority of the $\mathrm{PP}_{75} / \mathrm{TPU}_{25}$ blends over the $\mathrm{PP}_{25} / \mathrm{TPU} 75$ blends, because of the higher content of rigid segments in the blends.
\end{abstract}

Keywords. Polymer blends; maleic anhydride-grafted polypropylene; abrasive wear; hardness.

\section{Introduction}

Blending of different polymers is seen as an effective way for providing low-cost solutions to the development of new polymers with superior characteristics in the fields of science and industry [1-4]. In engineering polymers, thermoplastic polyurethane (TPU) is a kind of linear segmented block copolymer that contains relatively hard and soft structures $[5,6]$. Polyolefins can be mixed with TPUs to reduce the cost of the TPU and improve the thermal and mechanical characteristics (elastic modulus, strength and hardness, chemical properties and processing performance). Also, TPUs can be blended with polyolefins to improve the polyolefin characteristics (tear and impact strength, abrasion resistance, flexibility, shock absorbance and adhesion properties). Polypropylene (PP) is a kind of polyolefin (vinyl polymer) with a hydrogen atom substituted by the methyl group $\left(-\mathrm{H}_{2} \mathrm{C}-\mathrm{CRH}-\right)_{n}$ and has good mechanical and chemical properties. PP is generally selected as the second blending phase because of its good rigidity, ease of processing, chemical resistance, hightemperature stiffness and high melting point $\left(T_{\mathrm{m}}\right)$ [7-9]. Previous studies proved that the addition of PP into TPU causes an improvement in the thermal and also mechanical properties of TPU [4]. Bajsić et al [1,4] investigated the effects of different blending ratios of PP/TPU on the mechanical properties of the blends, and showed that PP above $20 \mathrm{wt} \%$ affected the elastic modulus considerably. Additionally, they found the partial miscibility of PP with TPU because of the lack of interaction of interfaces in the polymers. Jia et al [10] found that the strength of the mixture of PP/TPU is reduced up to $60 \%$ TPU concentration, and increased above the $60 \%$ TPU content due to the morphological and structural alterations induced by phase inversion.

The multiphase structure of the mixtures affects the mechanical properties of the blends seriously [2]. For improving the characteristics of the blends, the use of coupling agents in the structure is a preference. Besides, PP/TPU mixtures are already incompatible due to the extra interfacial stresses and diversity in their polarities $[2,10,11]$. Therefore, the multiphase structure of the PP/TPU blends generally leads to low thermal and mechanical characteristics [3]. The incorporation of a coupling agent and its reaction in the mixture can minimize the interfacial stress between the PP and TPU phases [12]. The coupling agents cause finer dispersion which leads to a larger content of the interfacial region [5]. To date, many coupling agents like maleic anhydride-grafted PP (PP-g-MA), secondary and primary amine-functionalized PP (PP-g-NHR and PP-g-NH ${ }_{2}$, respectively) and ethylene-vinyl 
acetate (EVA) copolymer have been tested for the blends and found to show advantageous impacts on the characteristics of PP/TPU mixtures [5,7]. PP-g-MA has maleic anhydride branch chains of a polar structure and also a non-polar backbone. The incorporation of PP-g-MA into the blends shows significant impacts, for example, increase the polarity of PP and decrease the melt viscosity of PP. They both can enhance the compatibility between the PP and TPU phases [13]. Jia et al [14] found that PP-g-MA effectively upgraded the major mechanical characteristics (flexural and tensile strengths, elongation at break values) of PP/TPU (70/30 and 30/70) blends, and they also showed 7 (phr) as the optimum concentration for PP-g-MA.

Except for the above-mentioned properties (thermal, mechanical, etc.), to the best of our knowledge, there have been no studies on the wear characteristics of PP/TPU blends against a sand paper. In reality, there are only a few studies regarding the tribological behaviour of the blends of TPU [1519]. In this study, polymer mixtures of PP/TPU of different weight ratios have been produced, containing functionalized polymer PP-g-MA of different contents as a coupling agent. The blends have been prepared by melt-blending using a twin screw extruder and then processed in an injection moulder to form the required samples for evaluating the effect of the compatibilization and test parameters on the abrasive wear properties of the blends. Then, the Shore D hardness test was also conducted and the relationships between the above-mentioned behaviours were analysed on the basis of the findings. Finally, the failure mechanisms and the wear characteristics of the PP/TPU blends were identified and discussed. It is believed that the developed material has potential to find use in the wear-resistant parts like sporting goods, medical devices, drive belts, footwear, etc. because of its good balance properties like high strength and elasticity, good abrasive wear resistance and light-weight properties. After the study, certain advantages and some limitations of the blends will be pointed out with the expectation that it will pave the way for further research in the wear studies on PP/TPU blends using different coupling agents, which could have a substantial impact on the applications of stronger and flexible blends with low density.

\section{Experimental}

\subsection{Materials}

TPU (ester-based) with a density of $1.22 \mathrm{~g} \mathrm{~cm}^{-3}$ (ISO 1183), hardness of 95 (Shore A, ISO 868) and tensile strength at break of $52 \mathrm{MPa}$ (DIN 53504) was purchased from Covestro AG, Germany. PP with a density of $0.905 \mathrm{~g} \mathrm{~cm}^{-3}$ (ASTM D1505), melt flow rate (MFR) of $4.5 \mathrm{~g} / 10 \mathrm{~min}$ $\left(230^{\circ} \mathrm{C} / 2.16 \mathrm{~kg}\right.$, ASTM D1238), melting point (DSC) of $163^{\circ} \mathrm{C}$ (ASTM D3417) and tensile strength at break of $42 \mathrm{MPa}$ (ASTM D638) was chosen as the second material (Petkim AS, Turkey). Maleic anhydride-grafted polypropylene (PP-g-MA) with a density of $0.9 \mathrm{~g} \mathrm{~cm}^{-3}$, melt flow rate (MFR) of $150 \mathrm{~g} / 10 \mathrm{~min}\left(230^{\circ} \mathrm{C} / 2.16 \mathrm{~kg}\right.$, ASTM D1238) was used as a compatibilizer (ExxonMobil Co., USA).

\subsection{Production of polymer blends}

PP/TPU/PP-g-MA blends were produced by melt blending in a twin screw (co-rotating intermesh) extruder with a screw diameter of $D=16 \mathrm{~mm}$ and a screw length/diameter $(L / D)$ ratio of 40, as seen in figure 1 (Gulnar, Turkey). Melt blending is a combination of the thermal and mechanical processes, which produce a homogeneous mixture of different polymers. In contrast to other methods, the most important advantage of melt blending is the absence of any solvents, which may have harmful effects on the structure of the final product. Two compositions were prepared with PP/TPU weight ratios of 75/25 and $25 / 75$, and the composition of PP-g-MA was changed between 5 and 11 (phr), as seen in table 1. Prior to mixing, PP, TPU and PP-g-MA pellets were dried in a drying oven at

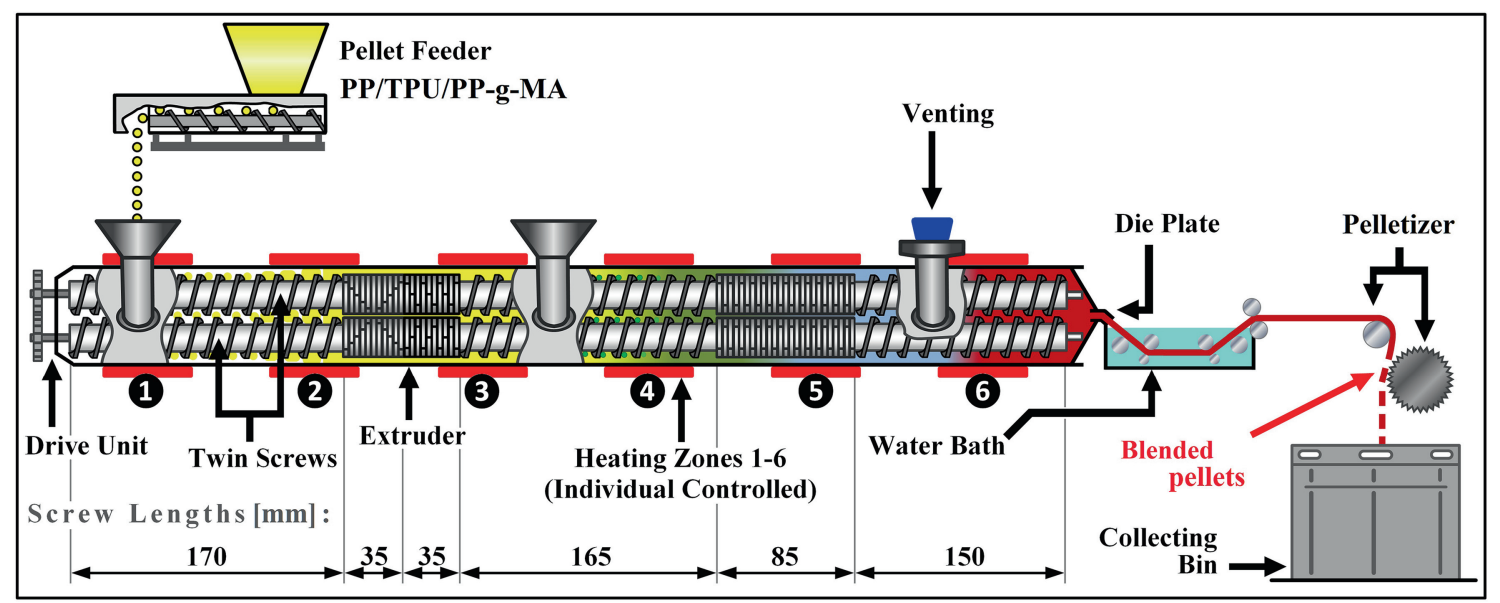

Figure 1. Schematic of co-rotating intermesh twin screw extruder used in this study. 
Table 1. Chemical composition of the samples used in this study.

\begin{tabular}{lccc}
\hline Sample code & PP (wt $\%)$ & TPU (wt $\%)$ & PP-g-MA (phr) \\
\hline PT5 & 75 & 25 & 5 \\
PT11 & 75 & 25 & 11 \\
TP5 & 25 & 75 & 5 \\
TP11 & 25 & 75 & 11 \\
PP & 100 & 0 & 0 \\
TPU & 0 & 100 & 0 \\
\hline
\end{tabular}

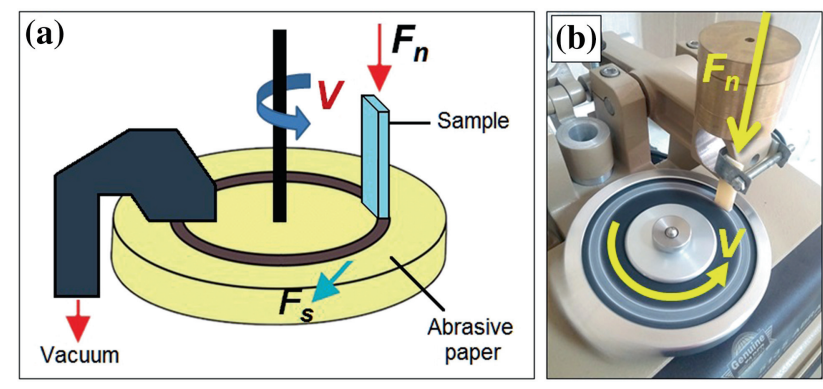

Figure 2. (a) Schematic diagram and (b) a photograph of the pinon-disc test rig.

$80^{\circ} \mathrm{C}$ for $24 \mathrm{~h}$ to remove moisture. The temperature profile of six zones of the extruder was controlled at 50, 195, 200, 200,195 and $190^{\circ} \mathrm{C}$, respectively, from the feeding zone to the heating zones. The melt compounded rod extrudate was then cooled by passing through a room-temperature water bath and granulated in situ using a pelletizer followed by drying in a drying oven at $80^{\circ} \mathrm{C}$ overnight. Before moulding, all polymer pellets were mixed with a spoon to form a homogeneous mixture. The pellets were then injection moulded into rectangular prisms wear test samples $\left(3 \times 12 \times 50 \mathrm{~mm}^{3}\right)$ by using a $12 \mathrm{ml}$ microinjection moulder (Xplore IM 12, The Netherlands). The barrel and mould temperatures were 205 and $25^{\circ} \mathrm{C}$, respectively, and the injection pressure was 7 bar. The pure polymers (PP and TPU) were also injection moulded to make blank control samples.

\subsection{Wear tests}

Wear tests were carried out using a Taber wear tester (Taber 5135, USA) as a pin-on-disc tribometer under dry sliding conditions against sand paper. A schematic representation of the wear test rig is shown in figure $2 \mathrm{a}$, and a photograph of the system is given in figure $2 \mathrm{~b}$. Contrary to the conventional pin/ball-on-disc tribometers where the rotating disc was placed in the horizontal position, the sample can be loaded horizontally by a cantilever mechanism against the vertically rotating disc for reducing the wear debris in the track (i.e., third-body effect), which changes the wear mechanism and the wear rate [20]. Similar studies on different materials that used such a test rig can be found in the literature [21-23]. In this study, the stationary sample was loaded vertically by a cantilever mechanism against the horizontally rotating disc, but the test rig was equipped with a vacuum system. The main feature of the configuration is to eliminate or reduce the trapping wear debris in the wear track, which alters the wear properties mentioned above. Samples, about $3 \times 12 \times 50 \mathrm{~mm}^{3}$, were polished one side on a $3 \times 12 \mathrm{~mm}^{2}$ surface with silicon carbide abrasive papers and fitted on a sample holder. The counterparts used in the tribo-tests were $\varnothing 102 \mathrm{~mm}$ abrasive sandpaper with a grit number of 150. Applied normal loads were 2 and $4 \mathrm{~N}$, and the sliding speeds in the centre of the wear track were 0.267 and $0.320 \mathrm{~m} \mathrm{~s}^{-1}$ (corresponding to the disc speeds of 60 and $72 \mathrm{rpm}$, respectively). Sliding distances were kept at 128 and $153.8 \mathrm{~m}$ for the disc speeds of 60 and $72 \mathrm{rpm}$, respectively (corresponding to a test duration of $8 \mathrm{~min}$ ). The track diameter was fixed at $85 \mathrm{~mm}$ in all the tests. Tribo-tests were performed at room temperature $\left(22 \pm 2^{\circ} \mathrm{C}\right)$ in atmosphere (relative humidity: $30 \pm 2 \%$ ). Before the tests, the samples were cleaned with ethyl alcohol and dried with clean compressed air. After the tribo-tests, the weight losses were measured as wear rates using a balance with $10^{-4} \mathrm{~g}$ sensitivity (Ohaus Pioneer, USA), and the morphology of the worn surface of the samples was observed using field emission scanning electron microscopy (FESEM, Zeiss Gemini 500, Germany). The samples were coated with a thin film of Au in a small sputtering chamber to make them conductive. Shore D hardness tests (Bareiss HPE II, Germany) were also conducted to see the relationship between the mechanical and wear properties of the samples. Eight hardness measurements were taken on each sample and the values were averaged.

\subsection{Design of the experiment method}

This method is an effective way to optimize a complex test, which helps to gain knowledge about the optimal levels using a large number of parameters [24]. A factorial design is important for calculating the overall interactions and the main effects of various test parameters. When using a full-factorial design analysis, every setting of every parameter appears with every setting of every other parameter. The most widespread design of the experiment is the one with all input parameters set at two levels each. The levels are classified as +1 and -1 or high and low, respectively. When the number of factors is $z$ with each at two levels, a full-factorial design represents $2^{z}$ cycles [25]. In this study, four factor, two level $\left(2^{4}=16\right.$ cycles) full-factorial design was used to investigate the tribological performance of the PP/TPU blends. Four factors, the TPU concentration, PP-g-MA content, normal load and sliding velocity, were selected to characterize the wear properties of the samples. Two levels were selected for each parameter. MINITAB Release 14 statistical software was selected for analysing the test data to determine the effect of various parameters and their interactions on the wear 
Table 2. Levels of the factors for L16 full-factorial experimental design.

\begin{tabular}{llcc}
\hline Symbol & Factor/parameter & Level 1 & Level 2 \\
\hline A & TPU concentration (\%) & 25 & 75 \\
B & PP-g-MA content (phr) & 5 & 11 \\
C & Normal load (N) & 2 & 4 \\
D & Sliding velocity (rpm) & $60^{\mathrm{a}}$ & $72^{\mathrm{b}}$ \\
\hline
\end{tabular}

${ }^{\mathrm{a}}$ Corresponding to a peripheral velocity of $0.267 \mathrm{~m} \mathrm{~s}^{-1}$.

${ }^{\mathrm{b}}$ Corresponding to a peripheral velocity of $0.320 \mathrm{~m} \mathrm{~s}^{-1}$.

Table 3. Experimental results for L16 full-factorial experimental design.

\begin{tabular}{|c|c|c|c|c|c|}
\hline \multirow{2}{*}{$\begin{array}{l}\text { Experimental } \\
\text { number }\end{array}$} & \multicolumn{4}{|c|}{$\begin{array}{l}\text { Parameters and } \\
\text { levels }\end{array}$} & \multirow{2}{*}{$\begin{array}{c}\begin{array}{c}\text { Experimental } \\
\text { results }\end{array} \\
\text { Wear loss }(\mathrm{g})\end{array}$} \\
\hline & A & $\mathrm{B}$ & $\mathrm{C}$ & D & \\
\hline 1 & 1 & 1 & 1 & 1 & 0.0491 \\
\hline 2 & 1 & 1 & 1 & 2 & 0.0811 \\
\hline 3 & 1 & 1 & 2 & 1 & 0.1811 \\
\hline 4 & 1 & 1 & 2 & 2 & 0.2631 \\
\hline 5 & 1 & 2 & 1 & 1 & 0.0487 \\
\hline 6 & 1 & 2 & 1 & 2 & 0.0543 \\
\hline 7 & 1 & 2 & 2 & 1 & 0.1620 \\
\hline 8 & 1 & 2 & 2 & 2 & 0.2689 \\
\hline 9 & 2 & 1 & 1 & 1 & 0.0665 \\
\hline 10 & 2 & 1 & 1 & 2 & 0.1243 \\
\hline 11 & 2 & 1 & 2 & 1 & 0.3006 \\
\hline 12 & 2 & 1 & 2 & 2 & 0.3306 \\
\hline 13 & 2 & 2 & 1 & 1 & 0.0724 \\
\hline 14 & 2 & 2 & 1 & 2 & 0.0866 \\
\hline 15 & 2 & 2 & 2 & 1 & 0.2632 \\
\hline 16 & 2 & 2 & 2 & 2 & 0.3566 \\
\hline
\end{tabular}

properties using 'the smaller the better' formulation given in equation (1) below:

$$
\frac{S}{N}=-10 \log \frac{1}{n}\left(\sum y^{2}\right),
$$

where $S / N$ is the signal-to-noise ratio, $n$ the number of observations and $y$ the respective characteristic. The parameters and the test values corresponding to their levels conducted in this study are shown in table 2. All 16 experiments were conducted in a random order for minimizing the effect of test errors on the wear test results.

\section{Results}

The experimental results for L16 full-factorial experimental design and wear loss values of pure polymers are given in tables 3 and 4, respectively. Pareto charts of the effects for wear loss are shown in figure 3a. A Pareto plot shows the absolute values of the effects of main factors and their interactions. A reference line indicates that the parameters
Table 4. Wear loss values of pure polymers.

\begin{tabular}{lccc}
\hline $\begin{array}{l}\text { Material } \\
\text { (pure) }\end{array}$ & $\begin{array}{c}\text { Normal } \\
\text { load (N) }\end{array}$ & $\begin{array}{c}\text { Sliding velocity } \\
(\mathrm{rpm})\end{array}$ & Wear loss (g) \\
\hline PP & 2 & 60 & 0.0311 \\
& 4 & 60 & 0.0925 \\
& 2 & 72 & 0.0448 \\
TPU & 4 & 72 & 0.1123 \\
& 2 & 60 & 0.0331 \\
& 4 & 60 & 0.0605 \\
& 2 & 72 & 0.0242 \\
& 4 & 72 & 0.0439 \\
\hline
\end{tabular}

(a)

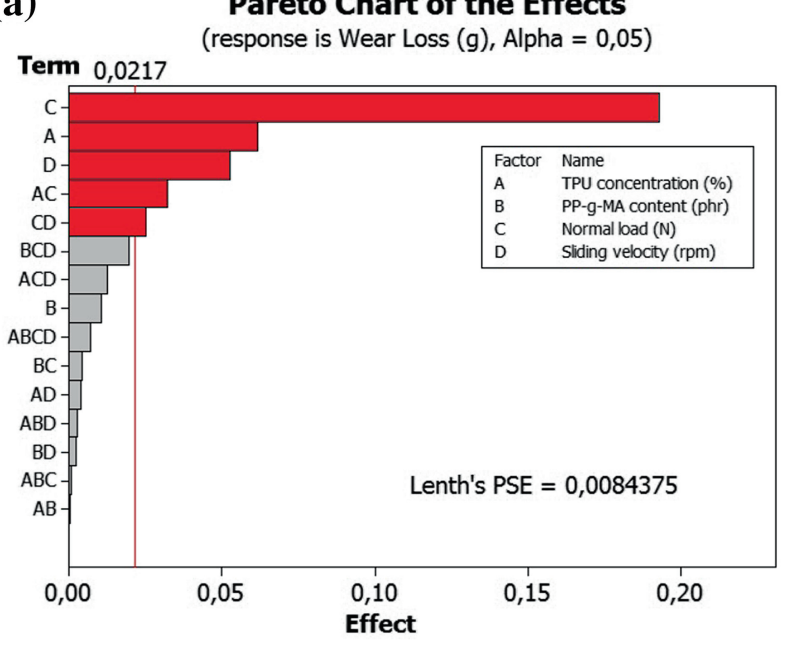

(b) Normal Plot of the Effects
Percent (response is Wear Loss $(\mathrm{g})$, Alpha $=0,05$ )

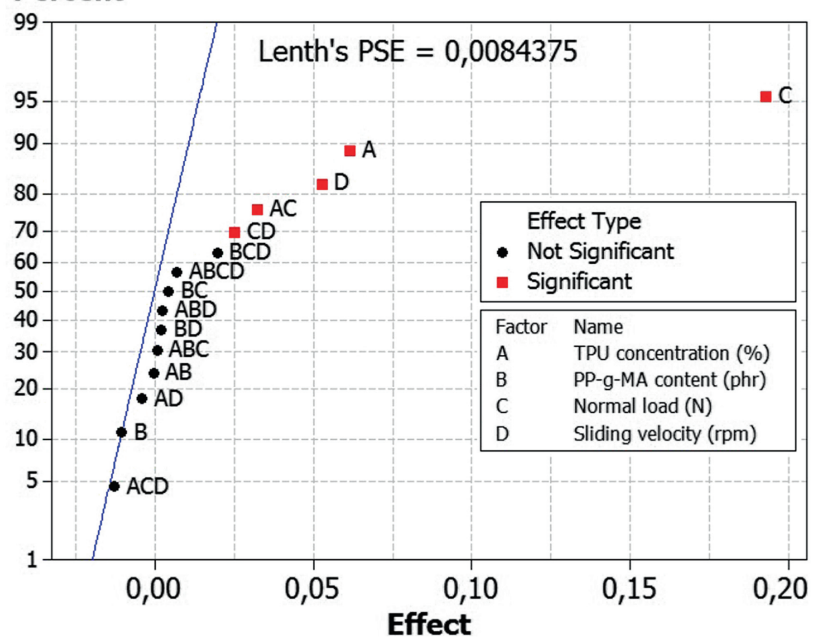

Figure 3. (a) Pareto chart and (b) normal plot of the effects for wear loss.

that extend past that line have relatively big importance. According to the wear test results, the factors that have the greatest effect on the wear rates are normal load, TPU concentration and sliding velocity, respectively, as seen in 
figure 3a. The theoretical basis of the plots is outside the scope of this study, but interested readers can find more information in ref. [26]. Furthermore, interactions of TPU concentration/normal load (AC), and normal load/sliding velocity (CD) were found potentially important. Similar graph to the Pareto charts is the normal plot of the effects, as seen in figure $3 \mathrm{~b}$. The effects further from zero are more statistically significant. The distance that must be points from zero to be statistically significant, depends on the significance level. From this plot, we can also conclude similarly that C, A, D, AC and CD appear to be significant.

As expected, the wear losses of pure PP and TPU were found to increase with an increase in the applied normal load, as seen in table 4. The wear loss of pure PP was also found to increase with an increase in the sliding velocity. But in the case of pure TPU, the opposite situation is observed. The wear loss of pure TPU was found to decrease with an increase in the sliding velocity. It is well known that TPU provokes 'stick-slip phenomena' initiated by the critical values of the sliding speed and normal load. It is generally characterized by drops of acoustic emission energy and peaks of friction coefficient [27]. It is thought that the decrease in wear loss is related to this phenomenon and also the soft structure of the pure TPU. The main effect plots for wear loss are shown in figure 4a. A main effect plot shows the relative importance of the effects of various parameters. A main effect is present when the mean response changes across the level of a parameter. The sign of the main effect shows the direction of the effect. As expected, the wear loss increases with the normal load and sliding velocity. The wear loss also increases with an increase in TPU concentration, and decreases with an increase in PP-g-MA content. The reasons are as follows; the segmented structure of TPU leads to high abrasion wear data, whereas PP consists of relatively long and entangled molecular chains which makes it more resistant to abrasion within the blends [15]. The TPUs have microphase separated domains composed of hard and soft segments (HSs and SSs, respectively). The HSs contain the diisocyanate components, and the SSs contain polyesters or polyethers $[1,4,28]$. The elastic modulus of the blends also tends to increase with the increase in rigid PP content in the blends [1]. In other words, an increase in the concentration of the ductile elastomer phase in a rigid polymer generally toughens the blend [14].

The interaction effect plots for wear loss are shown in figure $4 \mathrm{~b}$. The interaction effect plots show the mean response of various parameters at all possible combinations of their settings. When the plots are not parallel to each other, it is a proof of an interaction between the parameters. From the interaction effect plots for wear loss, as seen in figure $4 \mathrm{~b}$, no significant interaction was found between the factors.

SEM images of the worn surfaces of the blends and pure polymers after the wear tests are shown in figure 5. The worn surface of the samples is relatively rough compared with the unworn side surface of the samples. It is generally accepted that the most common wear modes of polymers are

\section{(a) Main Effects Plot for Wear Loss (g) Mean Data Means}
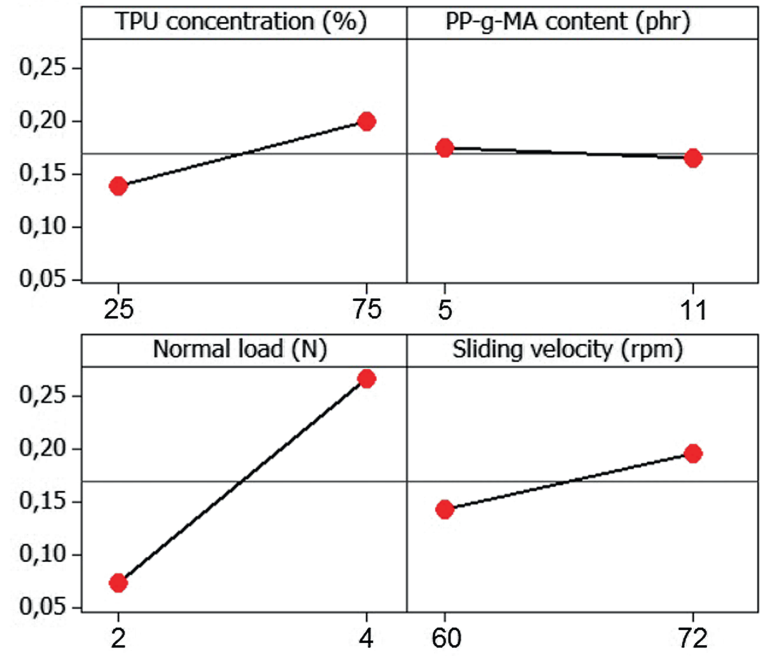

\section{(b) Interaction Plot for Wear Loss (g)} Data Means

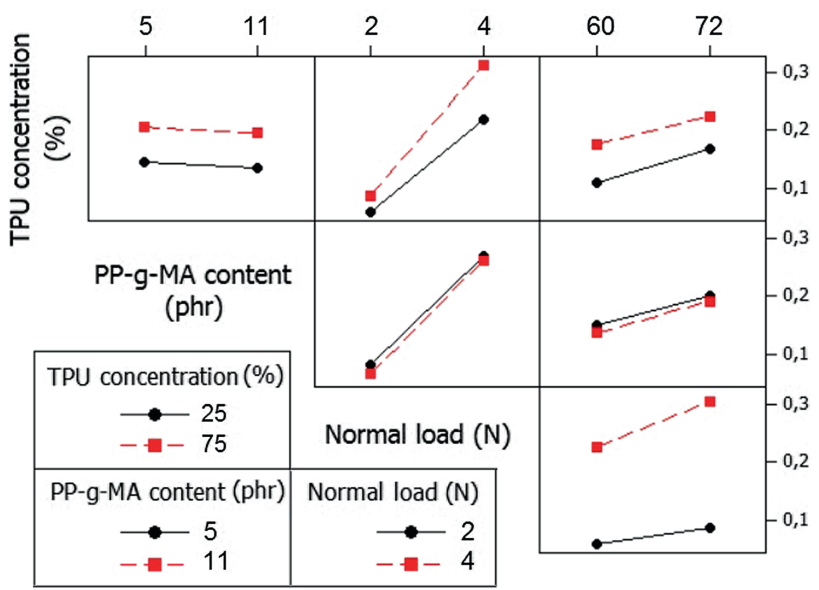

Sliding velocity (rpm)

Figure 4. (a) Main effect plots and (b) interaction effect plots for wear loss.

adhesion, abrasion and fatigue [29]. Also, it is well known that the wear rate of the polymers can be inversely proportional to the breaking stress and strain [30].

Although all blend worn surfaces show longitudinal scratches in macroscopic scale as a result of an abrasion mechanism, the wear mechanisms of plastic deformation and adhesive wear can also be seen in the track of pure PP as seen in the dashed square ' $\mathrm{A}$ ' marked in figure 5e. It appears that the TPU has become more susceptible to abrasive wear due to its soft structure (figure $5 \mathrm{f}$ ). Although wear mechanisms of the blends are among that of the pure polymers, no significant change has been observed with the compatibilizer content. The fatigue wave formation, which is common and associated with the fatigue mechanism due to the cyclic pass of asperity of the counterpart on the sample surface and can be assisted by the defects and a certain amount of stress 

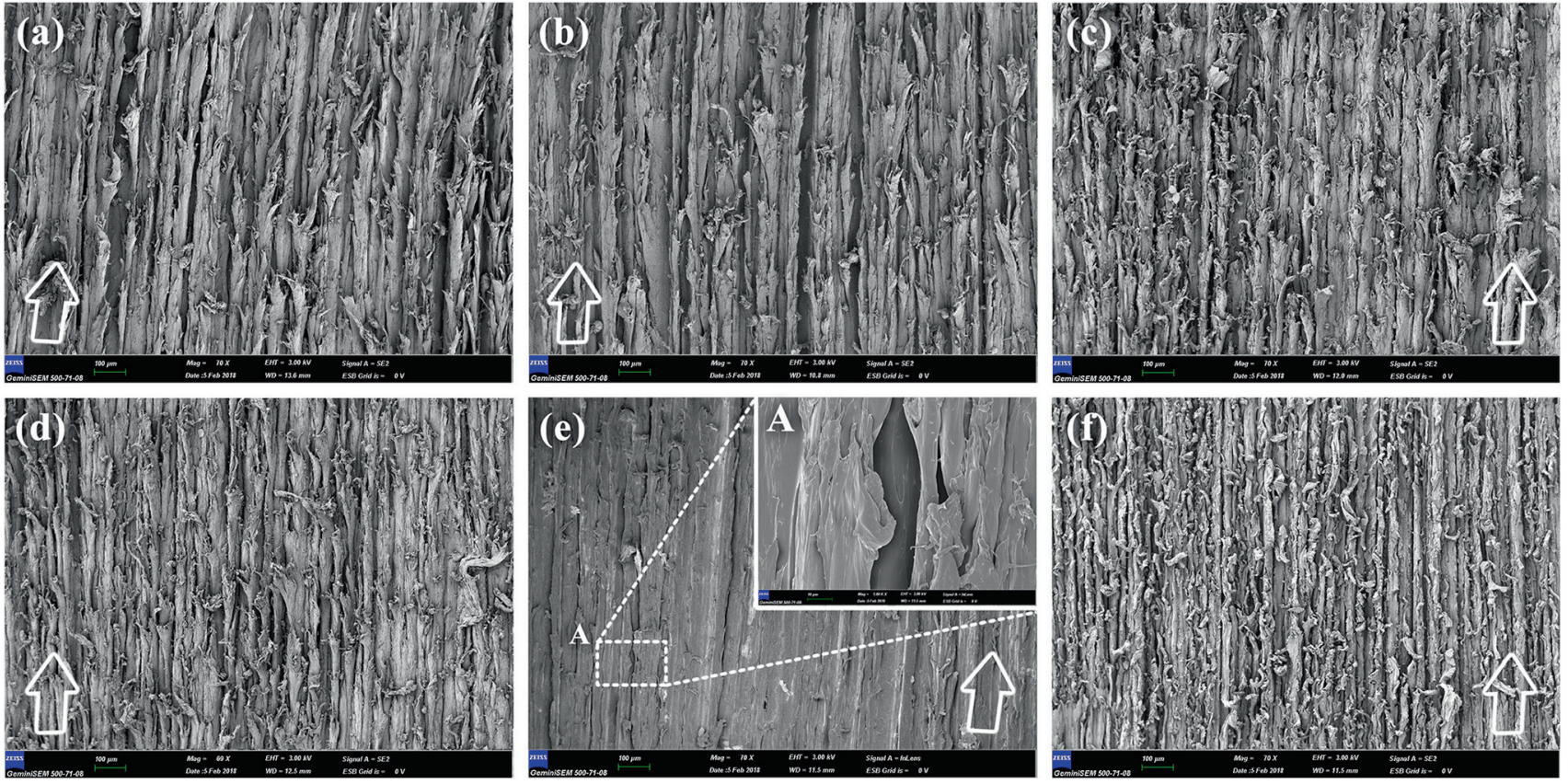

Figure 5. SEM images of the worn surfaces of the blends and pure polymers after the wear tests under the normal load of $4 \mathrm{~N}$ and sliding velocity of $72 \mathrm{rpm}$ : (a) $\mathrm{PP}_{75} / \mathrm{TPU}_{25} / 5 \%$ PP-g-MA, (b) $\mathrm{PP}_{75} / \mathrm{TPU}_{25} / 11 \%$ PP-g-MA, (c) PP $25 / \mathrm{TPU}_{75} / 5 \%$ PP-g-MA, (d) $\mathrm{PP}_{25} / \mathrm{TPU}_{75} / 11 \%$ PP-g-MA, (e) pure PP and (f) pure TPU (the blank arrows indicate the sliding direction of the abrasive paper).

concentration [28,29], was not observed in the worn surfaces of the samples. This is thought to be related to the abrasive nature of the sand paper.

The stuck wear debris particles were observed on the worn surfaces of the samples and also on the abrasive sandpapers despite the vacuum system of the test rig. The photographs of abrasive sand papers after the tribo-tests conducted for different parameters and $11 \%$ PP-g-MA concentration are seen in figure 6 . Some colour adjustments were made on the images to expose the stuck wear debris in the wear track. As seen in figure 6 , stuck wear debris in the wear track is broadening with increase in the normal load from 2 to $4 \mathrm{~N}$. Also, abrasive in sand papers used for $\mathrm{PP}_{25} / \mathrm{TPU}_{75}$ blends, exhibit a larger wear track width and trapped wear debris on the surface than those of $\mathrm{PP}_{75} / \mathrm{TPU}_{25}$ blends, because of the toughen effect of the soft TPU phase mentioned above. But, further investigation is required to assess the wear properties of the blends.

Shore D harness values of the PP/TPU/PP-g-MA blends and pure polymers are given in figure 7. It is expected that an increase in the amount of stiff polymer in an elastomer results in an increase in mechanical properties $[1,14]$. The TPU phase can toughen the PP, while the PP contributes to the modulus and strength. If the blends are well-harmonized, good synergies between the strength and the elongation at break can be achieved [5]. The blend morphology has an important influence in the reduction of properties. Blends with poor dispersity exhibit more reduction in useful properties [7]. Besides, the blend of the PP and TPU chains in a soft amorphous phase minimizes the mobility of the macromolecular polymer chains, which creates stiff PP/TPU blends when PP is

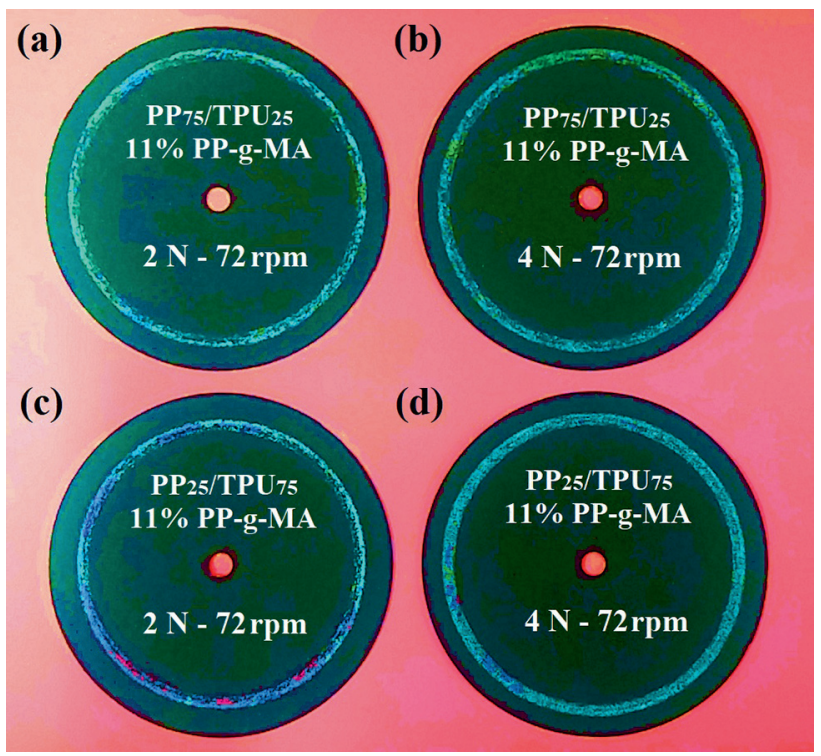

Figure 6. Photographs of abrasive sandpapers after the tribotests of different parameters against $(\mathbf{a}, \mathbf{b}) \mathrm{PP}_{75} / \mathrm{TPU}_{25}$ and $(\mathbf{c}, \mathbf{d})$ $\mathrm{PP}_{25} / \mathrm{TPU}_{75}$ blends.

the dominant phase [14]. Due to these facts, the $\mathrm{PP}_{75} / \mathrm{TPU}_{25}$ blends exhibit higher hardness and mechanical properties than $\mathrm{PP}_{25} / \mathrm{TPU}_{75}$ blends. Similar to the wear test results, the concentration of PP-g-MA compatibilization showed a small effect on Shore D hardness values of the blends. However, the hardness of the $\mathrm{PP}_{25} / \mathrm{TPU}_{75}$ blends increased from 53.51 to 


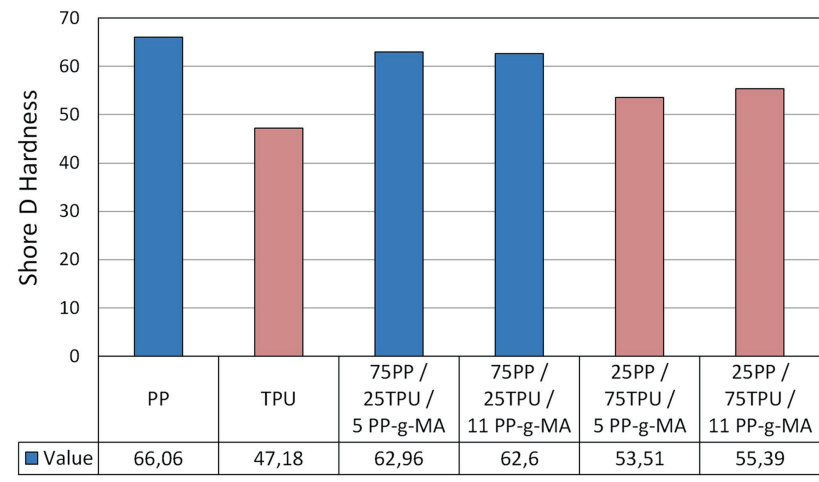

Figure 7. Shore D harness values of the PP/TPU/PP-g-MA blends and pure polymers.

55.39 Shore $\mathrm{D}$ with an increase in the PP-g-MA concentration from 5 to $11 \%$. Here, it is thought that it would be useful to carry out tests for blends with different concentrations to see the effect of the compatibilizer on dispersion of the clustered areas in the blends.

\section{Conclusions}

PP/TPU (75/25 and 25/75) blends with PP-g-MA (5 and 11 phr) were prepared successfully by melt blending and the effect of the coupling agent, blend weight ratios and test parameters on the wear properties of the blends were characterized. As expected, the test results showed the apparent superiority of the $\mathrm{PP}_{75} / \mathrm{TPU}_{25}$ blends over the $\mathrm{PP}_{25} / \mathrm{TPU}_{75}$ blends, because of the higher content of rigid segments in the blends. To put it another way, soft TPU in PP polymer by blending is not beneficial from the wear resistance point of view. The parameters that have the greatest effect on the wear loss are as follows; normal load, TPU concentration, sliding velocity and PP-g-MA concentration, respectively. The wear rate of the blends increases by increasing the normal load, TPU concentration, sliding speed, and slightly decreases by increasing the PP-g-MA concentration. Moreover, by combining the results of the mechanical and wear properties, the use of compatibilizer is seen to be a necessity to obtain highperformance blends, which are due to the good dispersion of the clustered areas within the blends.

\section{Acknowledgements}

This work was supported by the Office of Scientific Research Projects in Erciyes University, Turkey, under project no. FYL2017-7084.

\section{References}

[1] Bajsić E G, Šmit I and Leskovac M 2007 J. Appl. Polym. Sci. 1043980

[2] Di Y, Kang M, Zhao Y, Yan S and Wang X 2006 J. Appl. Polym. Sci. 99875

[3] Chuayjuljit S and Ketthongmongkol S 2012 J. Thermoplast. Compos. Mater. 26923

[4] Bajsić E G and Rek V 2004 e-Polymers 4-1 1

[5] Lu Q W and Macosko C W 2004 Polymer 451981

[6] Luo J-S, Xu B-P, Yu H-W, Du Y-X and Feng Y-H 2015 Fiber Polym. 1695

[7] Ajili S H, Ebrahimi N G and Khorasani M T $2003 \mathrm{~J}$. Appl. Polym. Sci. 892496

[8] Bajsić E G, Pustak A, Šmit I and Leskovac M $2010 \mathrm{~J}$. Appl. Polym. Sci. 1171378

[9] Kannan M, Joseph K and Thomas S 2015 Plast. Rubber. Compos. 44245

[10] Jia S, Qu J, Liu W, Wu C, Chen R, Zhai S et al 2014 Polym. Eng. Sci. $\mathbf{5 4} 716$

[11] Kannan M, Bhagawan S S, Joseph K and Thomas S 2009 J. Compos. Mater. 431915

[12] Pötschke P and Wallheinke K 1999 Polym. Eng. Sci. 39 1035

[13] Lan Y, Liu H, Cao X, Zhao S, Dai K, Yan X et al 2016 Polymer 9711

[14] Jia S, Zhu Y, Wang Z, Chen L and Fu L 2015 J. Polym. Res. 221

[15] Wang X, Mu B and Wang H 2015 Polym. Compos. 36897

[16] Chenglong W, Daoyuan P, Qibing W, Ruifang Z and Yuzeng Z 2014 Adv. Mat. Res. 915-916 193

[17] Poomali, Siddaramaiah, Suresha B and Lee J-H 2008 Mat. Sci. Eng. A-Struct. 492486

[18] Li B, Li M, Fan C, Ren M, Wu P, Luo L et al 2015 Compos. Sci. Technol. 10668

[19] Zhou S, Huang J and Zhang Q 2014 J. Thermoplast. Compos. 2718

[20] Devaraju A, Perumal A E, Alphonsa J, Kailas S V and Venugopal S 2012 Wear 28817

[21] Liu T, Wood W, Li B, Lively B and Zhong W-H 2012 Wear 294-295 326

[22] Prasad B K 2004 Wear 257110

[23] Zhang L C, Zarudi I and Xiao K Q 2006 Wear 261806

[24] Yu D, Wang C, Cheng X and Zhang F 2008 Appl. Surf. Sci. 2551865

[25] Gottipati R and Mishra S 2010 Chem. Eng. J. 16099

[26] Antony J 2003 Design of experiments for engineers and scientists (Burlington, MA: Butterworth-Heinemann)

[27] Yahiaoui M, Denape J, Paris J-Y, Ural A G, Alcalá N and Martínez F J 2014 Wear 315103

[28] Da Silva R C L, Da Silva C H and Medeiros J T N 2007 Wear 263974

[29] Myshkin N K, Petrokovets M I and Kovalev A V 2005 Tribol. Int. 38910

[30] Mathew M T, Novo J, Rocha L A, Covas J A and Gomes J R 2010 Tribol. Int. 431400 